\title{
ACTN3 R577X genotype is not associated with elite European Caucasian marathon performance
}

Adam J. Herbert*1, Alun G. Williams ${ }^{1,2}$, Sarah J. Lockey ${ }^{1,5}$, Robert M. Erskine ${ }^{2,3}$, Shane M. Heffernan ${ }^{1}$, Charles R. Pedlar ${ }^{4}$, Courtney Kipps ${ }^{2}$, Stephen H. Day ${ }^{1}$, Georgina K. Stebbings ${ }^{1}$

${ }^{1}$ Department of Exercise and Sport Science, Manchester Metropolitan University, UK.

(adam.herbert@stu.mmu.ac.uk, a.g.williams@mmu.ac.uk, shane.heffernan@stu.mmu.ac.uk, s.h.day@mmu.ac.uk,g.stebbings@mmu.ac.uk)

${ }^{2}$ Institute of Sport, Exercise \& Health, University College London, UK.

(c.kipps@ucl.ac.uk)

${ }^{3}$ Research Institute for Sport and Exercise Sciences, Liverpool John Moores University, UK. (r.m.erskine@ljmu.ac.uk)

${ }^{4}$ School of Sport, Health and Applied Science, St Mary's University, UK.

(Charles.pedlar@stmarys.ac.uk)

${ }^{5}$ School of Medical Education, Newcastle University, UK.

(sarah.lockey@ncl.ac.uk)

\section{Objectives}

A common nonsense polymorphism (R577X) in the ACTN3 ( $\alpha$-actinin-3 protein) has been associated with elite athlete status previously and, more specifically, the X-allele has been positively associated with elite endurance status. However, this remains inconclusive due to contradictory reports within the current literature. Thus, the current study aimed to compare ACTN3 R577X genotype and allele frequency distributions in 'elite' and 'sub-elite' marathon runners with those of a non-athletic, control population and to determine whether marathon personal best time was associated with ACTN3 R577X genotype.

\section{Method}

Four hundred and eighty four elite and sub-elite European Caucasian marathon runners and 554 ethnically matched controls provided a DNA sample from which the ACTN3 R577X polymorphism was genotyped using real-time PCR. Personal best (PB) times were used to determine elite (men $<2$ h 30 min, $n=111$; women $<3$ h 00 min, $n=105$ ) or sub-elite (men $2 \mathrm{~h}$ $30 \mathrm{~min}-2 \mathrm{~h} 45 \mathrm{~min}, \mathrm{n}=189$; women $3 \mathrm{~h} 00 \mathrm{~min}-3 \mathrm{~h} 15 \mathrm{~min}, \mathrm{n}=79$ ) status. Genotype and allele frequencies were compared between athletes and controls using Chi-square analyses. One-way ANOVAs were implemented to identify any genotype-dependent differences in PB times for men and women, which were subject to correction for multiple comparisons.

\section{Results}

The $\mathrm{X}$ allele was $\sim 3 \%$ more frequent in the marathon runners than in non-athlete controls (see Table 1 and Figure 1), although this small difference did not approach statistical significance. There were no significant differences in genotype $\left(\chi^{2}=3.40 ; P=0.182\right)$ or allele $\left(\chi^{2}=2.31 ; P\right.$ $=0.128)$ frequency distributions between athletes $(\mathrm{RR}=29.1 \%, \mathrm{RX}=50.6 \% \mathrm{XX}=20.2 \%$; $\mathrm{R}=54.4 \%, \mathrm{X}=45.6 \%)$ and controls. There were also no differences between elite and sub-elite genotype $\left(P=0.968, \chi^{2}=0.66\right)$ and allele frequencies $\left(P=0.916, \chi^{2}=0.11\right)$. Similarly, no differences in genotype or allele frequencies were found between either elite $\left(P=0.439, \chi^{2}=\right.$ $\left.1.65 ; P=0.265, \chi^{2}=1.24\right)$ or sub-elite $\left(P=0.254, \chi^{2}=2.74 ; P=0.183, \chi^{2}=1.772\right)$ runners and 
the control group. Neither were PB times genotype-dependent for either men $(P=0.864)$ or women $(P=0.966)$.

Table 1: ACTN3 R577X polymorphism count, genotype and allele frequencies in elite, subelite and control groups.

\begin{tabular}{llllll}
\hline & \multicolumn{3}{l}{ Genotype count (\%) } & \multicolumn{2}{l}{ Allele count (\%) } \\
\cline { 2 - 6 } & RR & RX & XX & R & X \\
Elite $(\mathrm{n}=216)$ & $64(29.6)$ & $108(50.0)$ & $44(20.4)$ & $236(54.6)$ & $196(45.4)$ \\
Sub-elite $(\mathrm{n}=268)$ & $77(28.7)$ & $137(51.1)$ & $54(20.1)$ & $291(54.3)$ & $245(45.7)$ \\
Controls $(\mathrm{n}=554)$ & $191(34.5)$ & $258(46.6)$ & $105(19.0)$ & $640(57.8)$ & $468(42.2)$ \\
\hline
\end{tabular}

\section{Conclusion}

No differences in genotype and allele frequencies were observed between athletes and controls, elite vs sub-elite, nor elite and sub-elite comparisons with the control group. Additionally, there was no genotype-dependent influence on PB time, which further emphasises that the ACTN3 R577X polymorphism does not influence elite endurance athlete status or determine marathon performance in European Caucasian runners. This is congruent with some previous findings and suggests other genetic variants or environmental factors may play a more prominent role in achieving elite endurance athlete status.

Keywords: ACTN3 R577X, endurance, marathon, performance. 